\title{
Review on Development and Application of Blended Learning
}

\author{
Wang Jingwen \\ Xi’an Fanyi University, Xi’an, Shaanxi, China, 710105
}

Keywords: leaning community; online and offline; self-paced learning

\begin{abstract}
Blended learning is recognized as the greatest trend occurred in higher education today. This paper reviews the research development of blended learning from the aspect of concept interpretation, BL's environment and technology, online and offline comparisons, learner's characteristics and perceptions, and issues and challenges existing in blended learning. This paper draws the conclusion that future research will mainly focus on how to create effective and satisfied blended learning experience, how to involve students in the dynamic leaning community, and how faculty effects during the implementation.
\end{abstract}

\section{Introduction}

It is an increasing evidence that Internet information and communication technologies are transforming the way people live and thought. The rapid emergence of digital technologies has had a huge impact on the possibilities for learning in the distributed environment. There are demands for universities to "provide for a larger and more diverse cross-section of the population, to cater for emerging patterns on educational involvement which facilitate lifelong learning and to include technology-based practices in the curriculum"' (Hicks \& Reid,2001). The term "Blended learning” is being referred more and more frequently. More and more universities are get used to online/Blend learning in many courses offerings. It is recognized as the greatest trend occurred in higher education today (Young, 2002).

\section{Conceptual Interpretation}

The definition of Blended learning (BL) is both simple and complex. From a simple view, blended learning is the integration of classroom face-to-face learning experiences with online learning experiences. It integrating the strengths of synchronous (face-to-face) and asynchronous (text/video-based Internet) learning activities (Young, 2002). The intersection of the two archetypes depicts where blended learning systems are emerging. Online and offline learning complements each other, and promotes learning and application behavior. From the complex view, there is considerable complexity in its implementation with the challenges of combining all kinds of delivery media and instructional methods. Graham (2004) divided blended learning into 4 levels based on a variety of organizational levels. It is Activity level, Course level, Program level, Institutional level. Blended learning provide another way of engaging learners in learning and being able to connect to a broader communities when they are in a study process. Through utilizing Platform and software to improve professional development, leadership development training and the delivery of instruction and assessment of students' work is what blended learning focus on (Keengwe \& Kang, 2012). Communications technologies now allows us to have synchronous distributed interactions almost as the same level of efficiency as in the F2F environment by the wide range use of virtual communities, instant messaging, blogging, etc. Besides, Ann Toler (2015) pointed that the top five components of blended learning are classroom instruction, interactive web-based training, email communication, self-paced content, and threaded discussion.

An Online Learning Survey revealed that blended learning was expanding globally to the growth rate $46 \%$ or higher per year (Ann Toler, 2015). For one part, it is because of the limitation of funding and time constraints of the higher education. More importantly, it is because of blended learning 
combines "the best of both worlds" (Schlageret al. 2002). It is flexibility in time and place, self-paced of online resources, increase of interactions and effectiveness of interaction between peers and instructors (Chamberlin and Moon 2005). Statistics from survey of "blended learning best practices" showed that over $85 \%$ of organizations are using some form Blended learning for supporting the delivery of contents. More than $76 \%$ of the respondents saying BL was more effective comparison with traditional training (Keengwe \& Kang, 2013). Blended learning not only offers possibilities to create transformative environments but also offering a new challenge for universities' instructors to provide the necessary teaching presence therefore helping students develop more responsibility for studying.

\section{Studies of BL's Environment and Technology}

In the information era, communication technologies now allow us to have synchronous and asynchronous distributed interactions that occur in real-time with close to the same levels as in the F2F environment. It is not difficult to find that there is an increasing focus on facilitating human interaction in the form of computer-supported collaboration, virtual communities, instant messaging, blogging, etc. Additionally, there is ongoing research investigating how to make machines and computer interfaces more social and human.

Early studies of information systems and simulation courses found that push technologies that deliver content directly to users, function better compared with technologies that required students to access content themselves (Parikh \& Vera, 2002). This phenomenon partly explains why there are more extensive studies on such technologies and Web 2.0 applications in online education. Alani (2002) found that email-driven systems produced higher learning outcomes than multimedia systems. Extending the concept of push technologies, Cao, Crews, Lin, Burgeon and Jr. Nunn maker (2008) examined the impact of "virtual interaction", defined as student interaction with a rich media representation of an instructor who provided targeted lecture video clips when students clicked on various keywords. Using five different treatment groups with varying levels of learner control of the virtual instructor, they compared student learning and perceptions relative to classroom-based delivery. Although all groups had significant increases in learning performance, there were no significant differences between the groups. Based upon these findings, the authors suggested that blending classroom-based and virtual interaction may lead to more effective learning. In a study of push technology that was not instructor dependent, Santana, Sasidharan and Webster (2008) examined the extent that a learning platform could help undergraduates in IT skill development. They found that the use of positively reinforcing scripts provided at the beginning and at the midpoint of the study significantly predicted student learning. Finally, tools such as blogs and wikis are beginning to be examined as learning tools. An example of this is provided by Watson, Boudreau, York, Greiner and Wynn's (2008) discussion of graduate students' use of wikis and open source software to co-create a textbook on programming in XML.

Regardless of the instructional methods used, all college programs should show quality in their content and delivery (Mandernach, 2005). Therefore, curriculum technology programs should be mapped out properly, strategically, updated and improvements should be clear for all ongoing planning and implementation stages and evaluation of all new or improved programs (Welch, 2007). In order to ensure the quality of online courses, the support from key top leaders is needed, the leadership team at various levels at the university should make sure that the infrastructure needs are in place to accommodate or support blended learning programs (Hilliard\& Bowie, 2015) Government and society should provide needed resources to faculty, students and staff. The leadership team at the university should continue to update faculty and students' skills and knowledge regarding new and improved technologies. (Arbaugh \& Godfrey, 2009) 


\section{Online and Offline Comparisons}

Comparison studies of online, offline and blended courses were an important theme during the past decade. Early studies showed no differences between these delivery mediums in terms of exam performance (Piccoli, Ahmad, \& Ives, 2001; Sankaran, \& Bui, 2000), but some found significant differences in student attitudes toward the Internet as a delivery medium. Although they found no differences in overall scores between the two mediums, Sankaran et al. (2000) found that students who are more positive toward the medium to which they were assigned showed significantly higher score improvements than students in their class section having less positive attitudes toward that medium. But we need to notice that merely comparing performance scores in an online and a classroom-based course is not in accordance with insights now. In a subsequent study, Sankaran and Bui (2001) found that performance differences were attributable to student learning strategies and motivation rather than to the characteristics of the delivery medium. Piccolo et al. (2001) found that although students in the online class they studied had higher computer self-efficacy, they were less satisfied with their course experience than were their classroom-based counterparts. Subsequent comparison studies examined a broader variety of delivery formats and outcome measures and found differences in academic performance and participation behaviors. When comparing blended and classroom offerings of an undergraduate course, Bryant, Campbell and Kerr (2003) found that students in the blended course performed better on exams, but at classroom students performed better on group projects and activity-based assignments. Using mixed group modes of synchronous and asynchronous delivery methods in an MBA-level information systems course, Clouse and Evans (2004) found that the combination of asynchronous content delivery and synchronous chat session produced the poorest performance on discrete exam questions, but that the combination of face-to-face content delivery and asynchronous discussion produced much more improvement on open-ended exam questions.

Heckman and Annabi (2005) compared the transcripts of student group case discussions in online and face-to-face sections of a capstone course. They found that students in the online discussions tended to take much more responsibility for creating the cognitive and social environment than did their classroom-based counterparts, and were more likely to demonstrate higher order cognitive processes as a result. The online students in the study were much more likely to respond to each other after posting responses to the instructor, while classroom students tended to respond to the instructor only. Comparison study that also included blended learning environments. Webb, Gill and Poe (2007) compared a range of blends on the purely classroom purely online continuum in the delivery of a case-based course. Controlling for several student demographic and experience characteristics, they found that the blended course offerings scored higher perceptions of the learning process and course outcomes. Similar controls were adopted by Jones, Moeeni and Ruby (2008) in their study of a blended course on telecommunications, but they found only a moderately significant relationship between the delivery medium and knowledge gain scores. Conversely, Al-Sham-mari (2008) found that web-based collaboration was dramatically less effective for the development of knowledge-based skill than class-room-based tools in a process engineering course. He found that classroom-based role play exercises scored particularly well on skill development. Recent studies are beginning to take longitudinal and theoretically grounded approaches to comparing the delivery mediums. Kock, Verville and Garza (2010) recently found that although classroom-based students had higher learning outcomes at the midpoint, these differences were gone by the course's end. Doo Hun Lim, Michael L. Morris, and Michael L. Morris (2013) found that group and individual projects, discussion activities and class assignment are noted as the most effective activities for students' blended learning through the compare of learning outcomes and other instructional variables between online and blended delivery methods. 


\section{Learner's Characteristics and Perceptions}

The studies of examine the relationships among participant characteristics, behaviors and course outcomes has come seize the public's attention. Student characteristics are mainly referred to personality, learning styles, and cultural orientation. The preliminary results of such studies suggest that conscientious, creative, and compliant students perform well in online courses, but extroverted students tend to have poorer performance (Kim \& Schniederjans, 2004; Schniederjans \& Kim, 2005). However, although learning styles have not been found to greatly influence performance among students, but ethnicity may predict performance ( $\mathrm{Lu}, \mathrm{Yu}, \& \mathrm{Liu}, 2003)$. For cultural orientation, the potential impact of regional and cultural differences received additional support in Wresch, Arbaughand Rebstock's (2005) study of an MBA course that compared students from German and American universities. They found that German students were much less likely to participate in online discussions than were American students. Hornik and Tupchiy (2006) examined the impact of cultural characteristics of US. They conclude that individualists tended to use the delivery medium less than collectivists, and were more likely to be dissatisfied with the medium and perform poorly in the course.

For interaction part, Studies of behavioral characteristic of students in online courses have found that interaction with other participants has enhanced student performance and attitudes toward online learning (Cappel \& Hayen, 2004; Heckman \& Annabi, 2005; Yukselturk \& Top, 2006). Johnson, Hornik and Salas (2008) examined social presence as a system design feature and its influence on application-specific computer self-efficacy, perceived usefulness, and course interaction in an online undergraduate course. They found that social presence of online system predicted satisfaction with the course and the value of its content, but did not predict learning performance. Participant interaction predicted performance and satisfaction. Student self-efficacy and perceived usefulness of the system predicted perceived content value, satisfaction, and learning performance. Other system-related research have pointed that students' attitudes and behaviors also influenced course management system usage. Saade (2007) and Bahli (2005) tested a model that integrated motivation, learning goal orientation, and cognitive absorption and found that even though motivation and learning goal orientation could predicted students' attitudes toward the online system, cognitive absorption was the most important factor to predict actual usage.

\section{Issues and Challenges existing in Blended Learning}

Blended learning approach combines the advantages of face to face learning with online learning. Chamberlin and Moon (2005) Learner pointed that learners are more satisfied with BL in the aspect of time and place flexibility, the utilization of resources, and the effectiveness and interaction between instructors and peers. But there are still issues can't be ignored. Graham (2004) concluded the major issues exits in blended learning. (1) The role of face to face interaction. How to combine the live interaction with virtual, asynchronous network interaction to provide needed support to learner. (2) The role of learner choice and self-regulation. Compared with traditional learning, learners are incline to get lost in the massive resource of network, online learning requires more self-monitoring and self-regulating. The increased demand of teacher's guidance in online learning environment is a main problem. It is urgent to increase students' learning capabilities and maturity of self-regulation. (3) The framework for Support and Training. Teachers need change attitudes to accept blended approaches. Providing learners and instructors with technological skills. The matured framework of blended learning could be provided not only from the technological infrastructure perspective but also from the organizational perspective. (4) Balance between innovation and production. Actually, it is a issue of input and output. How to make blended learning cost-effective, not only enhance teacher's instructing efficacy but also improving students' learning quality. Finding an appropriate balance between innovation and production is a constant challenge. 


\section{Conclusion}

Blended learning is a progressive, systematic and effective manner to teach and to learn. It includes with students' more active learning and participating, breaks the limitation of time and spaces, and expands the coverage of benefit students. After the specific research of literature. From the opinion of mine, the future research direction is about, firstly, how to create effective and satisfied blended learning experience which suits with many diverse learner, create constructive environment that connect the advantage of convenient distributed environment and the social interaction. Secondly, how to involving students in the dynamic leaning community and keep connect with the others smoothly? Give students needed support that teachers and assistant could provide timely coaching, mentoring, and advisement. Lastly, it is about the management issues. How faculty effects during the implementation of blended learning. How to integrate MOOC to Blended learning is a new research area too.

\section{Acknowledgement}

This work was financially supported by Xi'an Fanyi College, the construction and implementation of Blended learning's evaluation index system based on the training of practical personnel. (J17B29).

\section{References}

[1] Arbaugh, J. B., Godfrey, M. R., Johnson, M., Pollack, B. L., Niendorf, B., \& Wresch, W. (2009).

[2] Research in online and blended learning in the business disciplines: Key findings and possible future directions. The Internet and Higher Education, 12(2), 71-87.

[3] Bryant, K., Campbell, J., \& Kerr, D. (2003). Impact of web based flexible learning on academic performance in information systems. Journal of Information systems education, 14(1), 41-50.

[4] Chamberlin, S. A., \& Moon, S. M. (2005). Model-eliciting activities as a tool to develop and identify creatively gifted mathematicians. Prufrock Journal,17(1), 37-47.

[5] Garrison, D. R., \& Kanuka, H. (2004). Blended learning: Uncovering its transformative potential in higher education. The internet and higher education, 7(2), 95-105.

[6] Graham, C. R. (2006). Blended learning systems. CJ Bonk \& CR Graham, The handbook of blended learning: Global perspectives, local designs. Pfeiffer.

[7] Graham, C. R., \& Allen, S. (in press). Blended learning: An emerging trend in education. In C. Howard \& J. V. Boettecher \& L. Justice \& K. D. Schenk (Eds.), Encyclopedia of Distance Learning: Online Learning and Technologies (Vol. 4). Hershey, PA: Idea Group Inc.

[8] Heckman, R., \& Annabi, H. (2005). A content analytic comparison of learning processes in online and face-to-face case study discussions. Journal of Computer-Mediated Communication, 10(2), 00-00.

[9] Keengwe, J., \& Kang, J. J. (2012). Blended learning in teacher preparation programs: a literature review. International Journal of Information and Communication Technology Education (IJICTE), 8(2), 81-93.

[10] Kock, N., Verville, J., \& Garza, V. (2010). Media naturalness and online learning: Findings supporting both the significant-and no-significant-difference perspectives. Decision Sciences Journal of Innovative Education,5(2), 333-355.

[11] Hilliard, A. T. (2015). Global Blended Learning Practices for Teaching and Learning, Leadership, And Professional Development. Journal of International Education Research (JIER), 11(3), 179-188.

[12] Hicks, M., Reid, I., \& George, R. (2001). Enhancing on-line teaching: Designing responsive 
learning environments. International Journal for Academic Development, 6(2), 143-151.

[13] Israel, M. J. (2015). Effectiveness of Integrating MOOCs in Traditional Classrooms for Undergraduate Students. The International Review of Research in Open and Distributed Learning, 16(5).

[14] Jones, K. R., Moeeni, F., \& Ruby, P. (2008). Comparing web-based content delivery and instructor-led learning in a telecommunications course. Journal of Information Systems Education, 16(3), 265. 